\title{
Accurate Prediction of Cancers Stem Cells Incident using Enhanced Adaboost Algorithm
}

\author{
Mohamed Abd Elhamid Abbas, PhD \\ Dept. of Electrical Engineering, Faculty of Eng. \\ King Khaled Univ., KSA
}

\begin{abstract}
Cancers may abide or cycle after treatment because a brief aboriginal adjunct cells, bawled cancer stem cells, abides back to seed new tumors. Albeit scientists are not yet absolute about the bestowal cancer stem cells amusement in disease, apocalypse is acquiring that these cells are accurately antagonistic to chemotherapy and detachment, and can continue in the body deflates after treatment. Because cancer stem cells, which can cause new tumors, may endure backside after chemotherapy and radiation treatments, detecting aqueducts to aim these cells characteristically may allot a behavior to breed treatment accrual arrogant. But gaining access and analyzing cancer stem cells has been braving due to very minimal are convince in tumors and they are adverse to act and adjure alien the body. The halfway idealist of this paper is to portend the steps of cancer stem cells incident more precise and aptly. This paper decline a decipherment of adaboost algorithm to be adroit to foreshadow the concurrent steps of the cancer stem cells cardinal points. The enhanced version receives from stide detector its thoughts. The main merit of stide detector is its capability to predict the concurrent processes based on the space consumption complexity. The results show that there are clear divergence and convergence in error rate values of training and testing stages. And to obtain a precise prediction from the proposed algorithm, the threshold values should be in an average value. The results point to the proposed method is able to reduce the error rate at weak classifier number and high number training samples. Bottom of Form
\end{abstract}

\section{General Terms}

Stem Cells, Cancer, Prediction Algorithms

\section{Keywords}

cancer stem cells, adaboost algorithm, Stide detector, Data mining prediction, medical algorithms.

\section{INTRODUCTION}

Cancer stem cells , CSCs, are cancer cells, originate inside tumors, that own individuality allied with typical stem cells, particularly the aptitude to bestow mount to every cell types originate in a exacting cancer model. Charles A. Goldthwaite in [1] mention the characterization of CSCs will likely play a role in the development of novel targeted therapies designed to eradicate the most dangerous tumor cells. Detailed statistics are presented in [2,3]. Andrew $\mathrm{P}$ et al in [4] mention that cancer has a fundamentally common basis that is grounded in a polyclonal epigenetic disruption of stem/progenitor cells and more details about epigenomics of cancer are presented in [5]. Slamon DJ et al in [6] Concluded that Trastuzumab increases the clinical benefit of first-line chemotherapy in metastatic breast cancer that overexpresses HER2. Silvestri GA et al in
[7] review the mechanism of action, indications, contraindications, patient selection, and efficacy and side effects of this new class of compounds. Sherbenou DW et al in [8] applied the discovery of the Philadelphia chromosome. Hedge SR et al in [9] present systemic and targeted therapy for advanced colon cancer and some implications for the progression and treatment of metastatic disease are presented in [10]. The cancer stem cell theory elucidates not only the issue of tumor initiation and development but also the ineffectiveness of conventional cancer therapy $[11,12]$. Clarke MF et al in [13] focus on the investigation of cancer stem cells that offers the possibility of generating novel targets that could overcome issues of drug resistance, improve therapeutic efficacy. Rapp UR in [14] represent the relationship between Oncogene-induced plasticity and cancer stem cells.

A cell initiating human acute myeloid leukaemia after transplantation into SCID mice and Leukemia stem cells are introduced in $[15,16]$. Bonnet $\mathrm{D}$ et al in [17] describe Human myeloid leukemia is organized as a hierarchy that originates from a primitive hematopoietic cell. Prospective identification of tumorigenic breast cancer cells , A human colon cancer cell and Identification of human brain tumor initiating cells are presented in [18-20].In addition an identification of pancreatic cancer stem cells is presented in [21]. Hermann PC et al in [22] describe the distinct populations of cancer stem cells determine tumor growth and metastatic activity in human pancreatic cancer. Stem-cell abundant proteins Nanog, Nucleostemin and Musashi1 are highly expressed in malignant cervical epithelial cells as introduced in [23]. In addition there are number of researches in the fields of stem cell lines, Induction of pluripotent stem cells, Tumor dormancy and cancer stem cells, cancer stem cells in metastasis, Stem cells from regeneration to tumor formation, dormancy of solitary cells after successful extravasation and limited survival of early micrometastases are introduced in [24-29].

A launch formulas for the predictable morals allied with the chance that, initial from a solitary CSC, a specified number of CSC is reached, besides the predictable time it will obtain to accomplish that state. Prearranged inhabitants of unvarying size NSC, with M0 CSC at time $\mathrm{t}=0$.The replica of Embryonic Stem Cells are on hand in [30]. They presented the likelihood that just the once, the inhabitants will have M1CSC, around prearranged by:

$$
P\left(M_{0}, M_{1}\right)=\frac{1-r^{-M_{0}}}{1-r^{-M_{1}}}(1)[30]
$$

The universal appearance for the complex probability approved is given in their model as follows:

$$
P\left(M_{0}, M_{1}\right)=\frac{\theta\left(M_{0}\right)}{\theta\left(M_{1}\right)}(2)[30]
$$




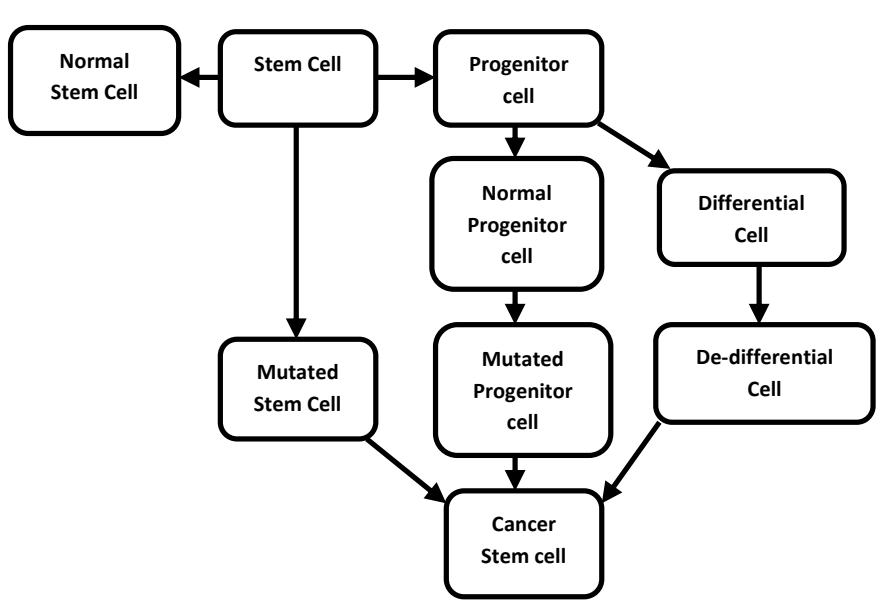

Figure 1 Cancer Stem Cells Incident [ 1 ]

The main objective of this paper is to predict accurately and efficiently the steps of cancer stem cells incident and the probability of its occurrence. This paper enhances a very high accurate algorithm called adaboost algorithm. The purpose of this enhancement is to add a necessary features of its training input suitable to deal the concurrent steps of cancer stem cells. After this enhancement we can use adaboost algorithm to obtain a more precise results of cancer stem cells Incident prediction. The rest of the paper is organized as follows: section 2 presents the previous works, section 3 initiates the proposed cancers stem cells prediction technique, section 4 pioneers the proposed algorithms of SCIPAS, section 5 begins the simulation results and a discussion about these results.

\section{RELATED WORK}

This section present previous work concerned with accurate prediction, clustering, statistical learning, association analysis, and link mining. This paper spots on two algorithmic techniques in prediction process: adaboost algorithm and stide detector. There are number of ensemble methods used to predict the behavior of the systems using multiple learners. But for simplicity we introduce only the two techniques used to achieve the paper objective.

\subsection{Adaboost Algorithm}

Adaboost Algorithm deals with schemes which utilize manifold learners to resolve a problem. The AdaBoost algorithm proposed by Yoav Freund and Robert Schapire is one of the most significant band methods, since it has concrete hypothetical groundwork, awfully precise forecast, enormous effortlessness, and broad and winning relevance. Let $\mathrm{X}$ indicates the case in point liberty and $\mathrm{Y}$ the set of class labels. Suppose $Y=\{-1,+1\}$. Specified a weak or base knowledge algorithm and a training set $\{(\mathrm{x} 1, \mathrm{y} 1),(\mathrm{x} 2, \mathrm{y} 2), \ldots,(\mathrm{xm}, \mathrm{ym})\}$ where $x i \in X$ and yi $\in Y(i=1, \ldots, m)$, the AdaBoost algorithm works as follows. Initial, it assigns equal weights to all the training examples (xi , yi $)(i \in\{1, \ldots, m\})$. Signify the allocation of the weights at the $t$-th learning encompassing as $\mathrm{Dt}$. From the training set and Dt the algorithm produces a weak or base learner ht $: \mathrm{X} \rightarrow \mathrm{Y}$ by vocation the base learning algorithm. Then, it uses the training instances to test ht, and the weights of the imperfectly classified examples will be augmented. Thus, an rationalized weight distribution $\mathrm{Dt}+1$ is attained. Commencing the training set and Dt+1 AdaBoost creates one more weak learner by vocation the base learning algorithm yet again [31].

\subsection{Stide detector}

Stide detector select the precise succession in two stages, training stage and testing stages[32]. A database consisting of templates of "self" is built from a watercourse of data careful to be regular; these are the training data. The stream is out of order into nearby, n-element, extend beyond subsequences, or $\mathrm{n}$-grams. These sole $\mathrm{n}$-grams are stored for future fast access. This completes the training stage. Each contrast in testing training obtaining a keep count of moreover zero or one. If the comparison is an exact match, the score is zero; if the contrast is not a competition, the score is one. These scores are summed within a restricted area to attain an irregularity signal. The Stide algorithm can be described formally as follows. Let $n$ be the length of a sequence. The similarity between the sequence $\mathrm{X}=(\mathrm{x} 0, \mathrm{x} 1, \mathrm{x} 2, \ldots \ldots \mathrm{xn}-1)$ and the sequence $\mathrm{Y}=(\mathrm{y} 0, \mathrm{y} 1$, $\mathrm{y} 2, \ldots \ldots, \mathrm{yn}-1)$, is defined by evaluating the similarities between $\mathrm{X}$ and $\mathrm{Y}$ by evaluating their space consumption complexity [32]. The valuation $\gamma(\mathrm{S})$ to predict the space consumption complexity of a sequence $S$ is calculated in equation (4)

$$
\gamma(s)=\frac{\operatorname{size}\left(\mathrm{H}_{4}^{\mathrm{S}}\right) / \operatorname{size}\left(H_{3}^{S}\right)}{\operatorname{size}\left(\mathrm{H}_{3}^{\mathrm{S}}\right) / \operatorname{size}\left(H_{2}^{S}\right)}=\frac{\operatorname{size}\left(H_{4}^{S}\right) \operatorname{size}\left(H_{2}^{S}\right)}{\operatorname{size}\left(H_{3}^{S}\right)^{2}}(4)
$$

Next section presents the proposed cancers stem cells incident prediction technique using both adaboost algorithm and stide detector

\section{PROPOSED CANCERS STEM CELLS PREDICTION TECHNIQUE}

The main objective of this paper is to propose an accurate prediction technique of cancer stem cells incident. In our proposed technique we used the main concept in stide detector in which it uses the space consumption complexity of concurrent training samples as an input of adaboost algorithm.

This new concept is expected to give the proposed technique more reliability in predicting cancer stem cells incident which depends on concurrent operations. This paper called the proposed prediction technique SCIPAS; the first initials of stem cells incident prediction using adaboost algorithm and stide detector. To start our prediction technique, stide detector using a similarity function to compare between space consumption complexity $(\gamma)$ and the normal data. It is important to declare a function called $\operatorname{sim}(\gamma, Y)$ back with zero if two progressions of the identical distance end to end are matching; or else the function returns one. Each cycle of size $\mathrm{N}$ in the test data is evaluated to each chain of size $\mathrm{N}$ in the regular database.

Given the set of space consumption complexity succession in the normal database, $\{\mathrm{y} 0, \mathrm{y} 1, \mathrm{y} 2,-----, \mathrm{y}(\mathrm{Norm}-1)\}$; for the efficient set of successions $\{\gamma 0, \gamma 1, \gamma 2,-----\gamma \mathrm{Z}-(\mathrm{N}-1)\}$ in the test data, where $\gamma \mathrm{s}=(\gamma \mathrm{s}, \ldots \ldots, \gamma \mathrm{S}+(\mathrm{N}-1))$ for $0 \leq \mathrm{s} \leq(\mathrm{Z}-(\mathrm{N}-$ 1)), and anyplace $Z$ is the numeral of elements in the data test. Then let $\gamma(\mathrm{S})$ denote the space consumption complexity and $\mathrm{Y}$ the set of class labels. Given a weak or base learning algorithm and a training set $\{(\gamma 1, \mathrm{y} 1),(\gamma 2, \mathrm{y} 2), \ldots,(\gamma \mathrm{m}, \mathrm{ym})\}$ where $\gamma$ i $€ \gamma(\mathrm{S})$ and yi $\epsilon \mathrm{Y}(\mathrm{i}=1, \ldots, \mathrm{m})$. We begin our modified formula of adaboost algorithm based on the equations and parameters in [30]. The space consumption complexity of embryonic stem cells is calculated in equation (5) using both of the equations (1) and (4).

$\gamma(s)=\frac{\theta\left(M_{2}\right)_{c t} \theta\left(M_{0}\right)_{c t}}{\theta\left(M_{1}\right)_{c t}^{2}}=\frac{\left(1-r^{-M_{2}}\right)_{c t}\left(1-r^{-M_{0}}\right)_{c t}}{\left(1-r^{-M_{1}}\right)_{c t}^{2}}(5)$ 
encompassing as Dt . From the training set and Dt the algorithm produces a weak or base learner ht $\gamma \rightarrow \mathrm{Y}$ by vocation the base learning algorithm. We can measure the error of Tw from equation 6 as go after:

$$
\mu_{t}=\sum_{i=1}^{N} P_{i}^{t}\left|T w\left(\gamma_{i}\right)-y_{i}\right|(6)
$$

Weight Distribution is useful in the field of human body capacity, using fraction degrees of the human body to conclude wherever a patient's weight is dispersed and what health risks this may compose . To settle on the weight of ht equation (7) should be computed as follow:

$$
\psi_{t}=\frac{1}{2} \ln \left(\frac{1-\mu t}{\mu t}\right)(7)
$$

A choice remnant is a engine learning replica consisting of a one-level decision tree. It is a decision tree with one interior node which is straight away associated with the terminal nodes. A choice remnant makes a forecast based on the cost of immediately a solitary input characteristic. Choice remnants are often used as workings called weak learners in machine learning band techniques such as carrier and enhancing. The next step is to funnel a weak learner ht from $\mathrm{S}$ using distribution St as depicted in equation (9) by the substituting the value of the space consumption complexity $\gamma i$ in equation(8).

$$
S_{t+1}(i)=\frac{S_{t}(i) \exp \left(-\psi_{t} y_{i} T w\left(\gamma_{i}\right)\right)}{N F}(8)
$$

$S_{t+1}(i)=\frac{S_{t}(i) \exp \left[-\psi_{t} y_{i} T w\left[\frac{\left(1-r^{-M 2}\right) \times\left(1-r^{-M 0}\right)}{1-r^{-M 1}}\right]\right]}{N F}(9)$ In the categorization duty the error rate is defined uncomplicatedly as the entitlement of the misclassified cases in the pragmatic in opposition to envisaged emergency data. The histogram of the error rate could be drawn using equation (10) as follow

$$
H s=\sum_{t=1}^{T} \psi_{t} T w\left[\frac{\left(1-r^{-M 2}\right) \times\left(1-r^{-M 0}\right)}{1-r^{-M 1}}\right](10)
$$

Where Tw : train a weak learner, $\mathrm{S}$ : the distribution of the weight, $\psi_{t}$ : the weight of ht $\boldsymbol{\mu}$ : error of ht, NF: the normalization factor and Hs: the histogram of the error rate. We can measure the efficiency of our proposed technique using the Error Rate formula of equation 10 . Next subsection presents our proposed SCIPAS technique used to realize our proposed prediction technique.

\section{PROPOSED ALGORITHMS OF SCIPAS}

This section presents the algorithms used to predict the cancer stem cells incident based on the space consumption complexity of stide detector and the accurate prediction of adaboost algorithm that depends on the space consumption complexity of cancers stem cells incident as an input. The main algorithm shown in table 1 depict that the space consumption complexity is calculated then the main program called the subroutine ADAC that predict actually the cancer stem cells incident using adaboost algorithm. ADAC, the first initials of adaboost algorithm based on space consumption complexity, is a subroutine with input data set represented with input data set represented by the space consumption complexity, base learning algorithm and number of learning rounds. The main process of the algorithm is to initialize the weight distribution and train a weak learner using this distribution. Then measure the error and finally determine the weight of the error.Table 2 depicts adaboost algorithm using Space Consumption Complexity. Next section commences the simulation results of the proposed algorithms and a discussion about these results.

Table 1. main algorithm of SCIPAS

\section{Begin ADAC}

Input: Let Data Set

$$
S=\left\{\left(\gamma_{1}, y_{1}\right),\left(\gamma_{2}, y_{2}\right), \ldots,\left(\gamma_{m}, y_{m}\right)\right\}
$$

Base learning algorithm $\mathrm{L}$

Number of learning rounds $\mathrm{R}$

Process:

//Initialize the weight distribution

S1(i) $=1 / \mathrm{m}$

For $\mathrm{t}=1, \ldots \ldots, \mathrm{R}$ :

$/ /$ Train a weak learner ht from $\mathrm{S}$ using distribution $\mathrm{St}$ $\mathrm{ht}=\mathrm{L}(\mathrm{S} 1, \mathrm{St})$;

//Measure the error ht

$$
\varepsilon_{t}=\operatorname{Pr}\left[h_{t}\left(\gamma_{i} \neq y_{i}\right)\right]
$$

//Determine the weight of ht

$$
\alpha_{t}=0.5 * \ln (1-\varepsilon t) /(\varepsilon t)
$$

$$
S_{t+1}(i)=\left(S_{t}(i) * \exp \left(-\alpha_{t} y_{i} h_{t}\left(\gamma_{i}\right)\right) / z t\right.
$$

End ADAC

Table 2 : Adaboost algorithm using Space Consumption Complexity

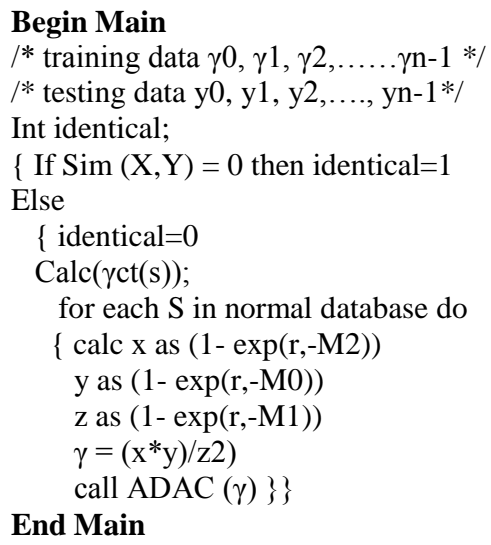

End Main

\section{RESULTS AND DISCUSSION}

The proposed technique is simulated using C\# Programming language. The training and testing error rates are computed using the new proposed concept where the error for a 
training example is the difference between the desired evaluation measure and the actual evaluation measure computed by the current state of the system. Figures [2-7] depict the relation between the weak classifier number and the error rate for both training and testing stages of the proposed algorithm. These figures consider the weak learner equal 10 and different training and testing samples. Figure 2 depicts the error rate in case of training and testing samples equal 50. The training and testing samples increases in figures from [3-7]. No of samples in figure 3 equals 100 , in figure 4 equals 200 , in figure 5 equals 300, in figure 6 equals 400 and in figure 7 equals 500. The results show that by increasing the number of trainings and testing samples the error rate of them go further to be identical.

Figures (8-12) depict the case of a fixed weak classifier number, training and testing samples at different values of the threshold. Figures (8-12) depict the error rate of training and testing stages are repeated after two fixed steps of increasing the threshold values. These results refer to the importance of fixing the threshold values during the proposed enhanced Adaboost algorithm to prevent the duplication of error values readings. Clearly figure 8 resembles figure 10 and figure 8 , despite the three figures are drawn at different values of threshold. The distance between the thresholds of the three figures are two steps fixed intervals. In addition this phenomenon exist in figure 9 and figure 11. Figures 8,10 and 12 depict a divergence and convergence in error rate values of training and testing stages. Figures 9 and 11 depict a clear divergence of these values. This go further to an important analysis, this analysis points to the fact that to obtain a precise prediction from the proposed algorithm, the threshold values should be in an average value. The threshold should not very high or very low. It is ended that there are multiple divergence and convergence in error rate. So, it is important to decrease the error rate by using a weak classifier number and choosing high number of samples in the training stage.

\section{CONCLUSIONS}

The sighting of Cancer stem cell in various tumor types has escorted in a new time of cancer investigation. Cancer stem cell discipline is a rising field that will eventually collision researchers' thoughtful of cancer courses and might classify new beneficial plans. The major idea of this paper is to offer a truthful forecast procedure of cancer stem cells occasion. This paper extend an edition of adaboost algorithm to be capable of foretelling the contemporaneous steps of the cancer stem cells incidence. The improved edition accepts from stide detector its contribution. The chief merit of stide detector is its competence to envisage the synchronized processes stand on the space expenditure complexity. The fallouts explain that there are obvious divergence and convergence in error rate principles of preparation and trying stages. To get hold of a clear-cut prophecy from the planned algorithm, the doorsill standards ought to be in a regular worth. The consequences spot the expected way is up to diminish the error rate at frail classifier number and soaring number training samples.

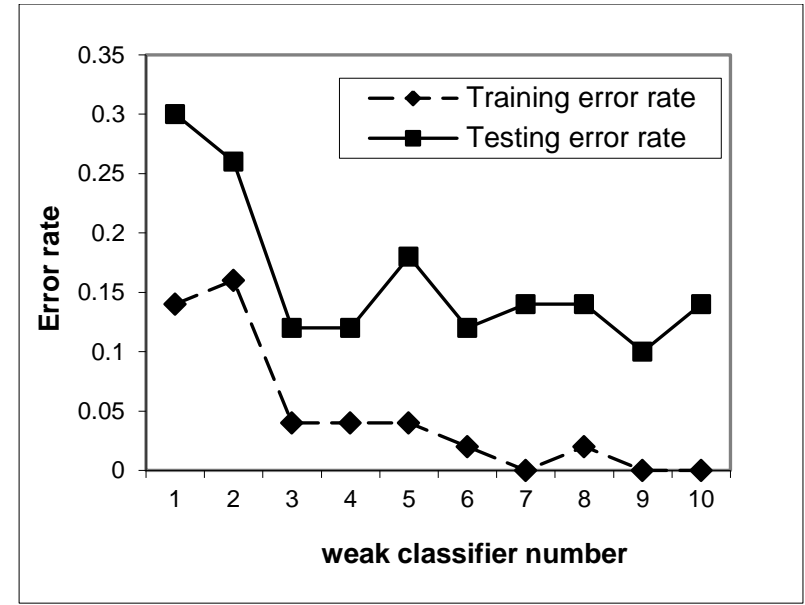

Figure 2 Error rate in case of training and testing samples equal 50

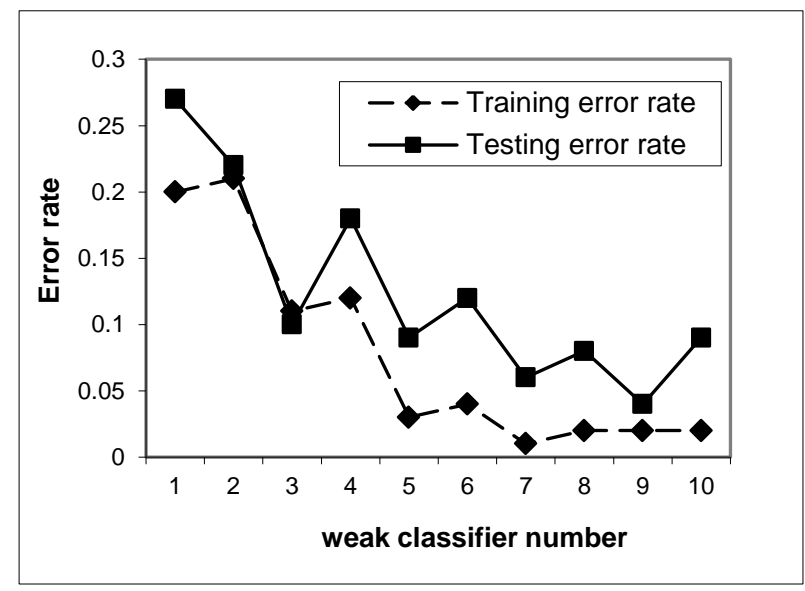

Figure 3 Error rate in case of training and testing samples equal 100

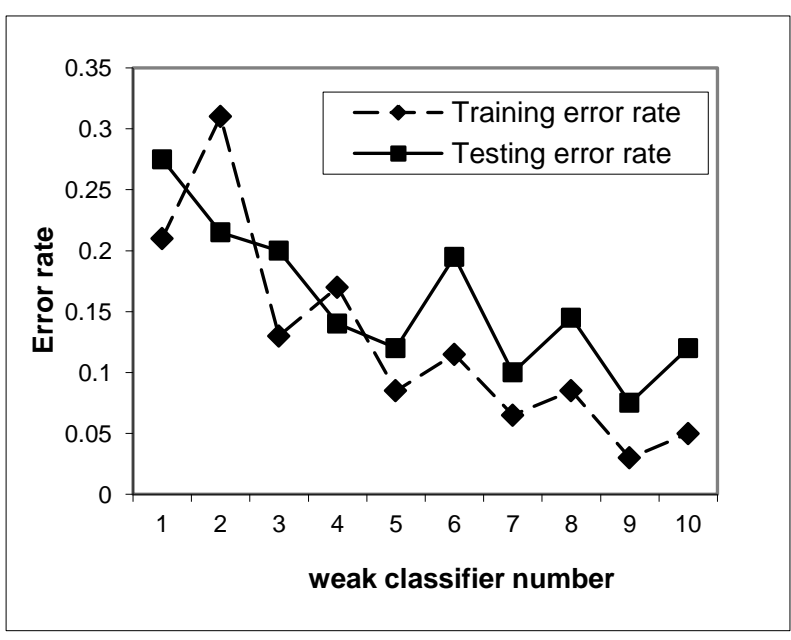

Figure 4 Error rate in case of training and testing samples equal 200 


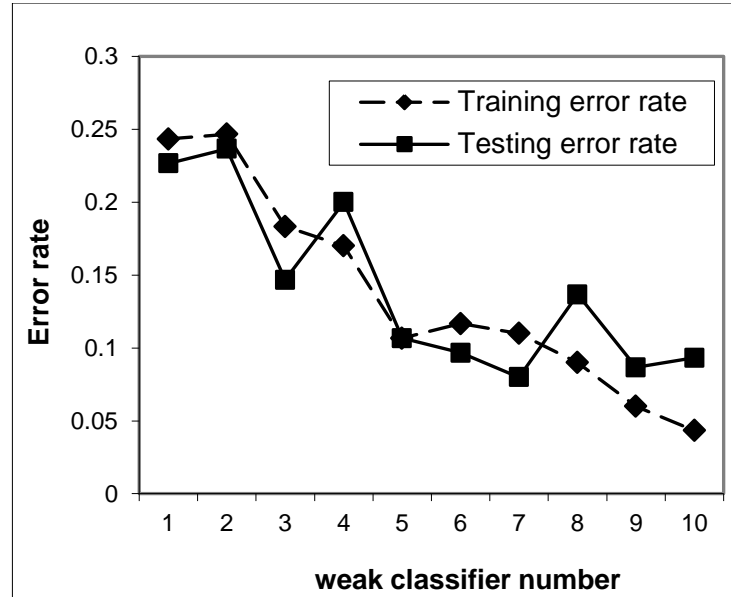

Figure 5 Error rate in case of training and testing samples equal 300

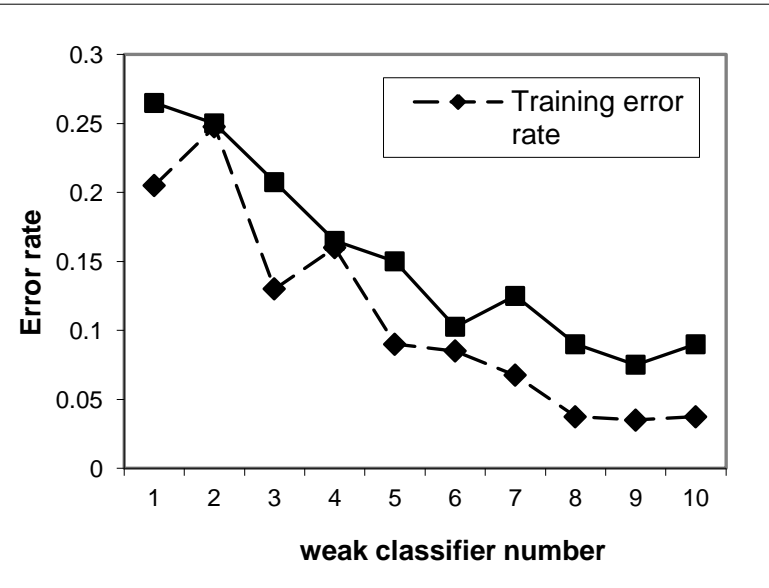

Figure 6 Error rate in case of training and testing samples equal 400

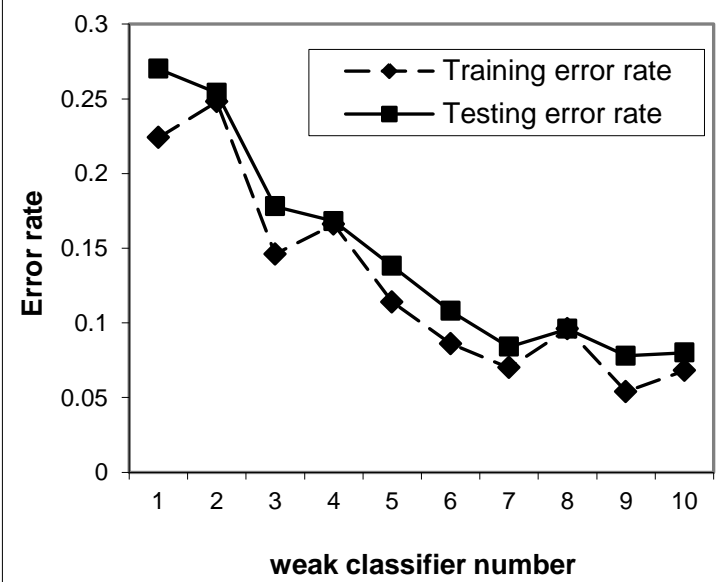

Figure 7 Error rate in case of training and testing samples equal 500

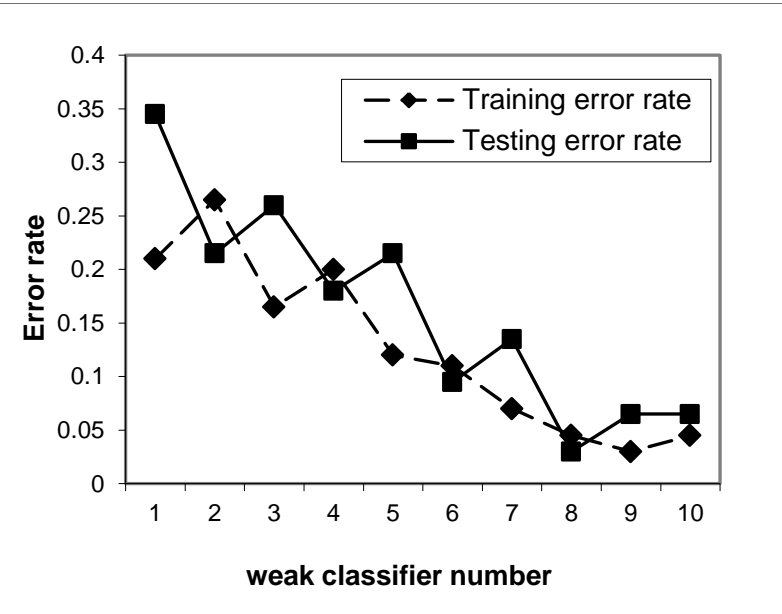

Figure 8 Error rate with threshold equal zero based on fixed samples and fixed weak learner

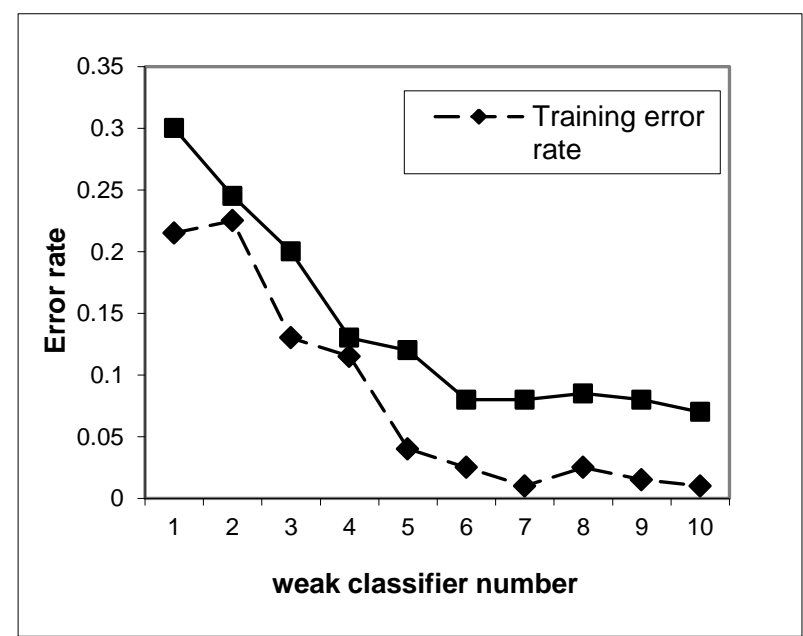

Figure 9 Figure 8 Error rate with threshold equal 0.1 based on fixed samples and fixed weak learner

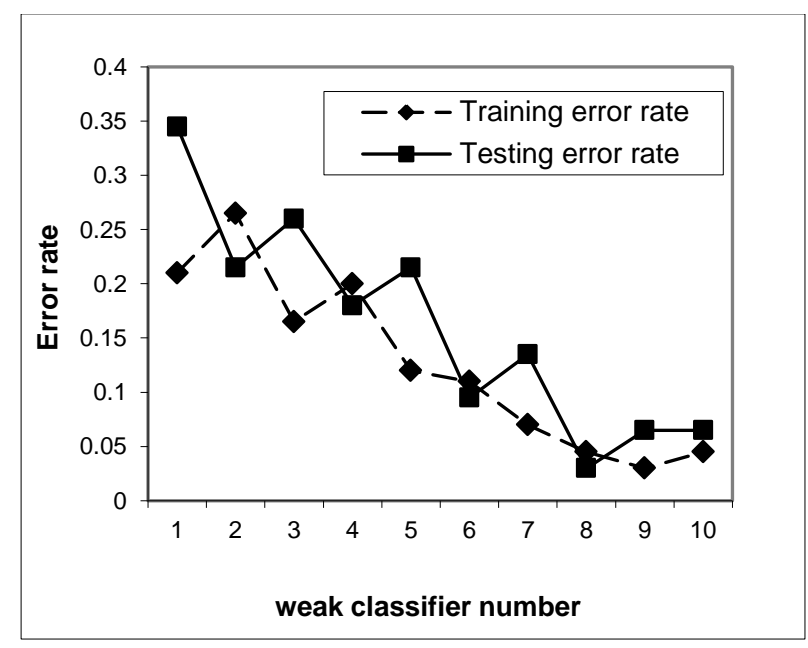

Figure 10 Figure 8 Error rate with threshold equal 0.2 based on fixed samples and fixed weak learner 


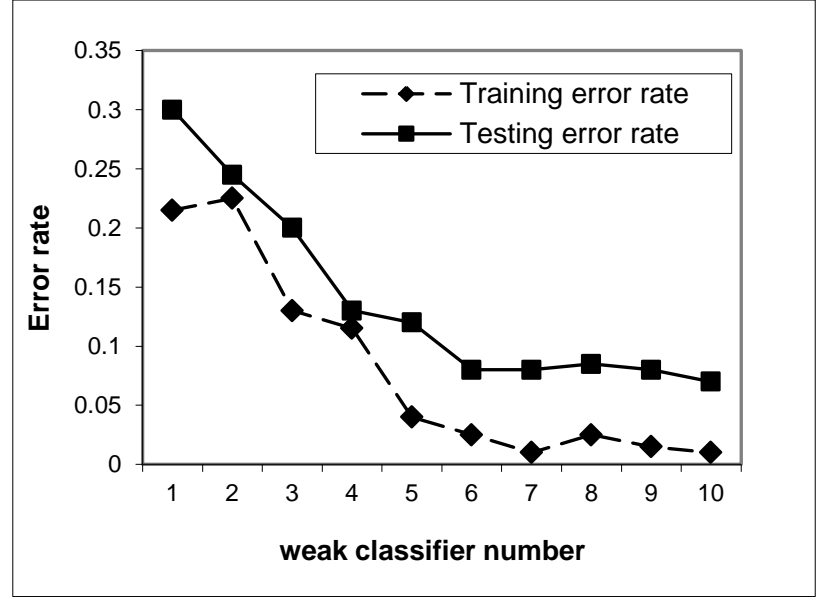

Figure 11 Figure 8 Error rate with threshold equal 0.3 based on fixed samples and fixed weak learner

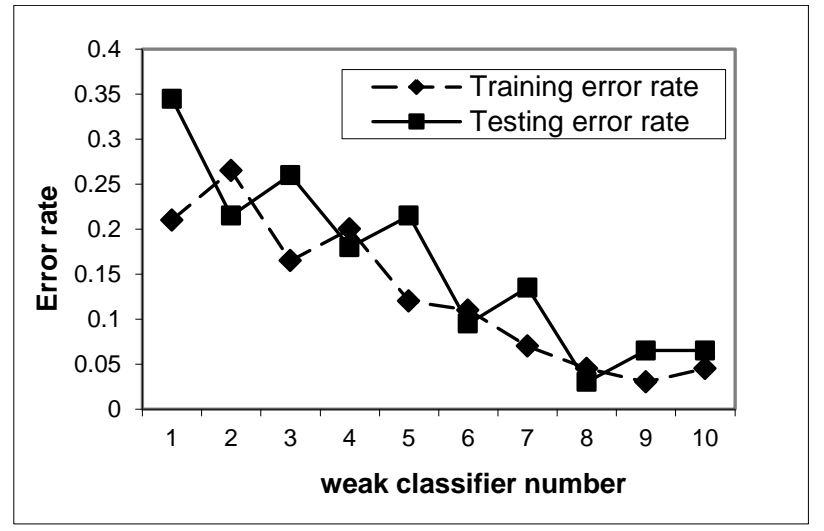

Figure 12 Figure 8 Error rate with threshold equal 0.4 based on fixed samples and fixed weak learner

\section{REFERENCES}

[1] Charles A. Goldthwaite, Jr., " Are Stem Cells Involved inCancer?",

http://stemcells.nih.gov/info/2006report/2006chapter9.htm , Accessed Jan. 22, 2012.

[2] National Cancer Institute. Surveillance Epidemiology and End Results: SEER stat fact sheets. http://seer.cancer.gov/data/. Accessed February 15, 2009.

[3] American Cancer Society. Cancer Facts \& Figures 2008. http://www.cancer.org/downloads/STT/2008CAFF finalsecured.pdf. Accessed February 15, 2009.

[4] Feinberg AP, Ohlsson R, Henikoff S. The epigenetic progenitor origin of human cancer. Rev Genet. 2006; 7:21-33.

[5] Jones PA, Baylin SB. The epigenomics of cancer. Cell. 2007;128:683-692.

[6] Slamon DJ, Leyland-Jones B, Shak S, et al. Use of chemotherapy plus a monoclonal antibody against HER2 for metastatic breast cancer that overexpresses HER2. N Engl J Med. 2001;344:783-792.

[7] Silvestri GA, Rivera MP. Targeted therapy for the treatment of advanced non-small cell lung cancer: a review of the epidermal growth factor receptor antagonists. Chest. 2005;128:3975-3984.
[8] Sherbenou DW, Druker BJ. Applying the discovery of the Philadelphia chromosome. J Clin Invest. 2007; 117:20672074.

[9] Hedge SR, Sun W, Lynch JP. Systemic and targeted therapy for advanced colon cancer. Expert Rev Gastroenterol Hepatol. 2008;2:135-149.

[10] Croker AK, Allan AL. Cancer stem cells: implications for the progression and treatment of metastatic disease. J Cell Mol Med. 2008;12:374-390.

[11] Gil J, Stembalska A, Pesz KA, Sasiadek MM. Cancer stem cells: the theory and perspectives in cancer therapy. J App Genet. 2008;49:193-199.

[12] Reya T, Morrison SJ, Clarke MF, Weissman IL. Stem cells, cancer, and cancer stem cells. Nature. 2001;414:105-111.

[13] Clarke MF, Dick JE, Dirks PB, et al. Cancer Stem CellsPerspectives on Current Status and Future Directions: AACR Workshop on Cancer Stem Cells. Cancer Res. 2006; 66:9339-9344.

[14] Rapp UR, Ceteci F, Schreck R. Oncogene-induced plasticity and cancer stem cells. Cell Cycle. 2008;7:45-51.

[15] Lapidot T, Sirard C, Vormoor J, et al. A cell initiating human acute myeloid leukaemia after transplantation into SCID mice. Nature. 1994;367:645-648.

[16] Huntly BJP, Gilliland DG. Leukemia stem cells and the evolution of cancer stem cells. Nat Rev Cancer. 2005;5:311-321

[17] Bonnet D, Dick JE. Human myeloid leukemia is organized as a hierarchy that originates from a primitive hematopoietic cell. Nat Med. 1997;3:730-737.

[18] Al-Hajj M, Wicha MS, Benito-Hernandez A, Morrison SJ, Clarke MF. Prospective identification of tumorigenic breast cancer cells. Proc Natl Acad Sci USA. 2003;100:3983-3988

[19] O'Brien CA, Pollett A, Gallinger S, Dick JE. A human colon cancer cell capable of initiating tumour growth in immunodeficient mice. Nature. 2007:445:106-110.

[20] Singh SK, Hawkins C, Clarke ID, et al. Identification of human brain tumor initiating cells. Nature. 2004 432:396-401.

[21] Li C, Heidt DG, Dalerba P, et al. Identification of pancreatic cancer stem cells. Cancer Res. 2007;67:10301037.

[22] Hermann PC, Huber SL, Herrler T, et al. Distinct populations of cancer stem cells determine tumor growth and metastatic activity in human pancreatic cancer. Cancer Stem Cell. 2007;1:313-323.

[23] Ye F, Zhou C, Cheng Q, Shen J, Chen H. Stem-cell abundant proteins Nanog, Nucleostemin and Musashi1 are highly expressed in malignant cervical epithelial cells. BMC Cancer. 2008;8:108.

[24] Yu J, Vodyanik MA, Smuga-Otto K, et al. Induced pluripotent stem cell lines derived from human somatic cells. Science. 2007;318:1917-1920.

[25] Takahashi K, Tanabe K, Ohnuki M, et al. Induction of pluripotent stem cells from adult human fibroblasts by defined factors. Cell. 2007;131:1-12. 
[26] Allan AL, Vantyghem SA, Tuck AB, Chambers AF. Tumor dormancy and cancer stem cells: implications for the biology and treatment of breast cancer metastasis. Breast Dis. 2007;26:87-98.

[27] Li F, Tiede B, Massague J, Kang Y. Beyond tumorigenesis: cancer stem cells in metastasis. Cell Res. 2007;17:3-14.

[28] Kucia M, Ratajczak MZ. Stem cells as a two-edged sword-from regeneration to tumor formation. J Physiol Pharmacol. 2006;57:5-16.

[29] Luzzi KJ, MacDonald IC, Schmidt EE, et al. Multistep nature of metastatic inefficiency: dormancy of solitary cells after successful extravasation and limited survival of early micrometastases. Am J Pathol. 1998;153:865-873.
[30] Katya Simeonova, Ganka Milanova, " Stem cell: from basic theoretical assumptions and mathematical concepts to the computational models", Simeonova and Milanova BMC Proceedings 2011, 5(Suppl 8):P108 http://www.biomedcentral.com/1753-6561/5/S8/P108.

[31] Xindong Wu • Vipin Kumar et al, " Top 10 algorithms in data mining", Knowl Inf Syst (2008) 14:1-37 DOI 10.1007/s10115-007-0114-2

[32] Roy A.Maxion and Kymic M.C. Tan, "Anomaly Detection in Embedded Systems" IEEE Transactions on computers,vol.51,No.2,pp.108-120 Feb.2002 